\title{
FUNDAMENTAL SKIN EFFECT IN ANISOTROPIC SOLID MECHANICS*
}

\author{
M. A. Biot \\ New York, N.Y.
}

\begin{abstract}
It is shown that in solid mechanics a skin effect is associated with anisotropy. Near a free surface or a surface of discontinuity cerlain components of the stress field vary rapidly from zero to a maximum within a thin skin. The thickness of this skin tends to vanish for increasing anisotropy. A stress concentration also occurs whereby certain stress components are amplified within the skin thickness. The analysis is carried out for a medium with or without initial stress and includes the case of surface instability, internal buckling and surface wave propagation. The results presented in the context of elasticity theory are on the whole valid for viscoelastic media by the principle of correspondence. The results are also applicable to multilayered or fibrous compound materials which, on the average, behave approximately as anisotropic continuous media.
\end{abstract}

\section{INTRODUCTION}

THE GENERAL equations used in the classical theories of deformation of anisotropic solids are very complex and involve many variables and elastic coefficients. As a consequence characteristic properties due to anisotropy and relevant parameters do not seem to have been fully recognized.

Our purpose here is to show that a basic feature in the mechanics of anisotropic solid is the appearance of a skin effect. The phenomenon referred to here will appear for example near a free surface where certain components of the stress field will vary very rapidly from zero to a maximum value within a thin skin. The thickness of the skin depends on the magnitude of the anisotropy and tends to zero when the anisotropy becomes very large. A skin effect will also occur near a rigid adhering boundary or in the vicinity of a surface of discontinuity.

Another phenomenon associated with the skin effect is a stress concentration whereby certain stress components within the skin thickness are magnified increasingly with increasing anisotropy.

In order to bring out these features in the simplest possible way, we have considered the restricted problem of plane-strain elasticity for an orthotropic incompressible solid. Some of the significant features due to finite deformation have also been included by applying the theory of elasticity of initially stressed solids.

The skin effect is analyzed in detail for three types of problems. The first is treated in Section 3 where the effect is derived for a surface under tangential loading. In Section 5 the skin effect is shown to appear in the buckling modes due to surface instability when a compressive stress is acting in a direction parallel to the surface. For internal buckling under initial stress the skin effect appears ncar a frce surface or a rigid adhering

* This work was supported by the AF Office of Scientific Research of the Office of Aerospace Research under Contract No. AF 49 (638)-1329. 
boundary as shown in Sections 6 and 7 .

It is remarkable that in all these cases the skin thickness is expressed by the same formula $\delta=\mathscr{L} / 2 \pi \sqrt{ }(2 m)$ where $\mathscr{L}$ is the wave length of the deformation along the surface of discontinuity and $m$ is a fundamental dimensionless parameter measuring the magnitude of the anisotropy.

The result is by no means restricted by the simplifying assumption of incompressibility. A brief discussion in Section 8 indicates that the same parameter $m$ determines the skin thickness for an anisotropic compressible medium provided we use for $m$ the value obtained in earlier theories in terms of the various elastic coefficients of the anisotropic solid. The generalization including compressibility is valid for initially stressed solids. As indicated in the last section, the principle of viscoelastic correspondence suggests further generalizations of these results to purely viscous and viscoelastic anisotropic solids.

While the skin effect becomes more significant with increasing anisotropy its importance is not confined to such cases. Actually the effect brings to light certain fundamental properties of elastic solutions which are already present for isotropic media. These properties provide a new outlook and suggest new methods of attack in many practical problems of "Strength of Materials."

Another important field of application of these results is the mechanics of thinly layered or fibrous structures where the anisotropy is due to the use of composite materials of strongly contrasting rigidities. By viscoelastic correspondence this includes the case of heterogeneous media composed of different viscoelastic materials.

\section{SIMPLIFIED EQUATIONS FOR ANISOTROPIC ELASTICITY}

Consider a plane-strain deformation in the $x, y$ plane. The material is assumed incompressible of orthotropic symmetry with axes of symmetry parallel to the $x, y$ axes, and initially stress-free.

The two-dimensional stress-strain relations of the material are

$$
\begin{aligned}
\sigma_{x x}-\sigma & =2 N e_{x x} \\
\sigma_{y y}-\sigma & =2 N e_{y y} \\
\sigma_{x y} & =2 Q e_{x y}
\end{aligned}
$$

with a condition of incompressibility

$$
e_{x x}+e_{y y}=0
$$

The usual stress components for plane strain are $\sigma_{x x} \sigma_{y y} \sigma_{x y}$ and the strain components are defined by

$$
\begin{aligned}
& e_{x x}=\frac{\partial u}{\partial x} \quad e_{y y}=\frac{\partial v}{\partial y} \\
& e_{x y}=\frac{1}{2}\left(\frac{\partial v}{\partial x}+\frac{\partial u}{\partial y}\right)
\end{aligned}
$$

where $u$ and $v$ are the displacement components in the $x, y$ plane. The plane-strain elastic properties are defined by the two elastic constants $N$ and $Q$. The significance of 
$\sigma$ is obtained by adding the first two of equations (2.1) taking into account relation (2.2). We find

$$
\sigma=\frac{1}{2}\left(\sigma_{x x}+\sigma_{y y}\right)
$$

Equations (2.1) are a particular case of the incremental stress-strain relations derived by the author for the initially stressed medium $[1,2]$. They are obviously applicable to a medium initially stress-free. Note that the left side of equations (2.1) represents a twodimensional stress deviator. In the case of anisotropy it is different from the threedimensional stress deviator as already pointed out $[1,2]$. We must add the condition of equilibrium of the stress field

$$
\begin{aligned}
& \frac{\partial \sigma_{x x}}{\partial x}+\frac{\partial \sigma_{x y}}{\partial y}=0 \\
& \frac{\partial \sigma_{x y}}{\partial x}+\frac{\partial \sigma_{y y}}{\partial y}=0
\end{aligned}
$$

The set of equations (2.1) to (2.5) is solved by introducing the function $\phi(x, y)$ and putting

$$
u=-\frac{\partial \phi}{\partial y} \quad v=\frac{\partial \phi}{\partial x}
$$

Elimination of all variables except $\sigma$ and $\phi$ yields

$$
\begin{aligned}
& \frac{\partial \sigma}{\partial x}-\frac{\partial}{\partial y}\left[(2 N-Q) \frac{\partial^{2} \phi}{\partial x^{2}}+Q \frac{\partial^{2} \phi}{\partial y^{2}}\right]=0 \\
& \frac{\partial \sigma}{\partial y}+\frac{\partial}{\partial x}\left[(2 N-Q) \frac{\partial^{2} \phi}{\partial y^{2}}+Q \frac{\partial^{2} \phi}{\partial x^{2}}\right]=0
\end{aligned}
$$

Finally elimination of $\sigma$ yields

$$
\frac{\partial^{4} \phi}{\partial x^{4}}+2\left(\frac{2 N}{Q}-1\right) \frac{\partial^{4} \phi}{\partial x^{2} \partial y^{2}}+\frac{\partial^{4} \phi}{\partial y^{4}}=0 .
$$

The plane-strain problem is now reduced to finding $\sigma$ and $\phi$ from equations (2.7) and (2.8).

We shall consider solutions which are sinusoidal along the $x$ direction. Such a solution of equations (2.7) and (2.8) is

$$
\begin{aligned}
& \phi=\frac{1}{l^{2}} f(l y) \sin l x \\
& \sigma=Q\left(m f^{\prime}-f^{\prime \prime \prime}\right) \cos l x
\end{aligned}
$$

where $f(l y)$ satisfies the differential equation

$$
f^{\prime \prime \prime \prime}-2 m f^{\prime \prime}+f=0 .
$$

The primes denote differentiation with respect to the argument $l y$ and we have put

$$
m=\frac{2 N}{Q}-1 \text {. }
$$


Stresses and displacements are now derived completely by means of a single unknown function $f$ in the following form

$$
\begin{aligned}
u & =U(l y) \sin l x \\
v & =V(l y) \cos l x \\
\sigma_{x x} & =r(l y) \cos l x \\
\sigma_{y y} & =q(l y) \cos l x \\
\sigma_{x y} & =\tau(l y) \sin l x
\end{aligned}
$$

with

$$
\begin{aligned}
l U(l y) & =-f^{\prime} \\
l V(l y) & =f \\
\frac{1}{Q} r(l y) & =-f^{\prime}-f^{\prime \prime \prime} \\
\frac{1}{Q} q(l y) & =(2 m+1) f^{\prime}-f^{\prime \prime \prime} \\
\frac{1}{Q} \tau(l y) & =-f-f^{\prime \prime} .
\end{aligned}
$$

These results provide complete solutions of a large variety of problems.

Basic solutions of the differential equation (2.10) are of the type

$$
f=\mathrm{e}^{\beta l y}
$$

where $\beta$ satisfies the characteristic equation

$$
\beta^{4}-2 m \beta^{2}+1=0 .
$$

In the present analysis we shall assume

$$
N>Q
$$

hence

$$
m>1
$$

The limiting case $N=Q, m=1$ corresponds to an isotropic medium. Under the assumptions (2.16) and (2.17) the four roots of the biquadratic equation (2.15) are always real. Two of the roots $\beta_{1}$ and $\beta_{2}$ are positive. They are

$$
\begin{aligned}
& \beta_{1}=\left[m+\sqrt{ }\left(m^{2}-1\right)\right]^{\frac{1}{2}} \\
& \beta_{2}=\left[m-\sqrt{ }\left(m^{2}-1\right)\right]^{\frac{1}{2}}
\end{aligned}
$$

the radicals being chosen positive.

These roots satisfy the conditions

$$
\beta_{1} \beta_{2}=1 \quad \beta_{1}^{2}+\beta_{1}^{2}=2 m
$$

The other two roots are the negative values $-\beta_{1}$ and $-\beta_{2}$. 
With four arbitrary constants $C_{i}$ the general solution of the differential equation $(\dot{2} .10)$ is

$$
f=C_{1} \mathrm{e}^{\beta_{1} l y}+C_{2} \mathrm{e}^{\beta_{2} l y}+C_{3} \mathrm{e}^{-\beta_{1} l y}+C_{4} \mathrm{e}^{-\beta_{2} l y} .
$$

\section{SKIN EFFECT UNDER TANGENTIAL SURFACE LOADS}

Consider the elastic half space occupying the region $y<0$ with the plane boundary $y=0$.

Solutions which vanish at $y=-\infty$ are obtained by putting $C_{3}=C_{4}=0$ in expression (2.20). Such a solution retains only two arbitrary constants and is written

$$
f=C_{1} f_{1}+C_{2} f_{2}
$$

with

$$
\begin{aligned}
& f_{1}=\mathrm{e}^{\beta_{1} l y} \\
& f_{2}=\mathrm{e}^{\beta_{2} l y}
\end{aligned}
$$

The salient feature in which we are interested in here is a consequence of the fact that if $N / Q$ is sufficiently large, hence for large anisotropy, the value if $\beta_{1}$ is large while $\beta_{2}$ is small. For example let us assume

$$
N / Q=4 \cdot 5
$$

From equations (2.18) and (2.19) we may write approximately

$$
\begin{aligned}
& \beta_{1} \cong \sqrt{ }(2 m)=4 \\
& \beta_{2} \cong \frac{1}{\sqrt{(2 m)}}=\frac{1}{4} .
\end{aligned}
$$

Hence

$$
\frac{\beta_{1}}{\beta_{2}}=16 .
$$

In this case although $N / Q$ is not really very large we see that $\beta_{2}$ may be considered small relative to $\beta_{1}$. Therefore the function $f_{1}$ decays much more rapidly than $f_{2}$ when we move away from the surface.

Skin effect

We choose values $C_{1}=-1, C_{2}=1$ for the constants in the solution (3.1). Hence

$$
f=f_{2}-f_{1} \text {. }
$$

We substitute this expression into equations (2.13). After taking into account relations (2.19) we find the following equations for $q$ and $\tau$

$$
\begin{aligned}
& \frac{1}{Q} q(l y)=\left(\beta_{1}+\beta_{2}\right)\left(f_{2}-f_{1}\right) \\
& \frac{1}{Q} \tau(l y)=\left(1+\beta_{1}^{2}\right) f_{1}-\left(1+\beta_{2}^{2}\right) f_{2} .
\end{aligned}
$$


At $y=0$ we find $f_{2}=f_{1}=1$ and $q=0$. Hence expressions (3.6) correspond to a solution for which the normal stress $\sigma_{y y}$ at the surface is zero. Because $\beta_{1} \gg \beta_{2}$ the value of the tengential stress $\tau$ is given approximately by

$$
\frac{1}{Q} \tau(l y)=\beta_{1}^{2} f_{1} .
$$

This value decays rapidly with depth as shown schematically in Fig. 1 . It decreases by a factor $1 / \mathrm{e}$ at a depth

$$
\delta=\frac{1}{\beta_{1} l}=\frac{\mathscr{L}}{2 \pi \beta_{1}}
$$

where $\mathscr{L}=2 \pi / l$ is the wavelength of the tangential surface load distribution. With the approximation (3.4) we may also write

$$
\delta \cong \frac{\mathscr{L}}{2 \pi \sqrt{(2 m)}} .
$$

The length $\delta$ represents a "skin thickness". It is proportional to the wave length and decreases approximately as the inverse square root of the "anisotropy ratio" $N / Q$. For $N / Q=4.5$ its value is

$$
\delta \cong \frac{\mathscr{L}}{25} .
$$

By substituting expression (3.6) for $f$ into the first two of equations (2.13) we may also derive the values of the displacements $U$ and $V$. At the surface we find $V=0$. Hence the tangential load produces no vertical displacement at the surface. We also obtain

$$
\tau(0)=2 \sqrt{ }(N Q) l U(0)
$$

where $\tau(0)$ and $U(0)$ are the tangential load and tangential displacement at the surface. Equation (3.12) agrees with earlier results $[1,3]$.

\section{Stress concentration}

Consider the normal stress distribution $\sigma_{x x}=r(l y)$ as a function of the depth at the abscissa $x=0$. Substitution of expression (3.6) into the third of equations (2.13) yields

$$
\frac{1}{Q} r(l y)=\beta_{1}\left(1+\beta_{1}^{2}\right) f_{1}-\beta_{2}\left(1+\beta_{2}^{2}\right) f_{2} .
$$

Hence for $\beta_{1} \geqslant \beta_{2}$ we write approximately

$$
\frac{1}{Q} r(l y)=\beta_{1}^{3} f_{1} .
$$

This value also decays rapidly with depth and exhibits the same skin effect as the shear stress $\tau$. In addition, comparison with the value (3.8) of $\tau$ leads to the relation

$$
r=\beta_{1} \tau
$$

This shows a stress concentration corresponding to a magnification of the shear stress $\tau$ by a factor $\beta_{1}$. This magnification increases approximately as the square root of the 
anisotropy ratio $N / Q$. The skin effect and the magnification of $\sigma_{x x}$ are illustrated in Fig. 1.

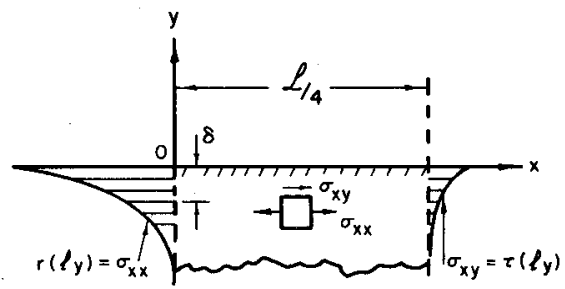

FIG. 1. Skin effect due to a tangential load distribution $\sigma_{x y}=t(0) \sin l x$ of wavelength $\mathscr{L}=2 \pi / l$ applied at the surface $y=0$. Distribution with depth of stresses $\sigma_{x x}$ and $\sigma_{x y}$ is shown respectively at $x=0$ and $x=\mathscr{L} / 4$. Values of $r(l y)$ and $\tau(l y)$ are both positive.

An important feature of this stress concentration is its independence from the wavelength.

\section{ANISOTROPIC ELASTICITY WITH INITIAL STRESS}

A similar skin effect is obtained in the case of an anisotropic solid with initial stress. The discussion is carried out as in the previous case by applying the theory of initially stressed anisotropic solids. $[1,2]$.

The anisotropy in this case may be "intrinsic" or "induced". What is meant is that the anisotropy may be present in the material initially in the stress-free state or it may be induced by the initial stress itself. In any case the anisotropy which we consider here refers to incremental properties. The principal directions of the initial stress as well as those of the elastic symmetry are assumed to coincide with the coordinate axes. For simplicity we shall again assume the medium to be incompressible. This implies relation (2.2).

The incremental stress-strain relations in this case, are $[1,2]$,

$$
\begin{aligned}
s_{11}-s & =2 N e_{x x} \\
s_{22}-s & =2 N e_{y y} \\
s_{12} & =2 Q e_{x y}
\end{aligned}
$$

where $s_{11} s_{22} s_{12}$ denote incremental stresses referred to locally rotated axes with incremental elastic coefficients $N, Q$. The local rotation is

$$
\omega=\frac{1}{2}\left(\frac{\partial v}{\partial x}-\frac{\partial u}{\partial y}\right)
$$

The elastic coefficients $N, Q$ will in general be functions of the initial stress.

Let the initial stress be reduced to a single principal component $S_{11}=-P$ in the $x$ 
direction. Because the medium is incompressible this does not restrict the generality since the addition of an initial hydrostatic stress has no effect on the deformation.

The equilibrium equations for incremental stresses are

$$
\begin{aligned}
& \frac{\partial s_{11}}{\partial x}+\frac{\partial s_{12}}{\partial y}-P \frac{\partial \omega}{\partial y}=0 \\
& \frac{\partial s_{12}}{\partial x}+\frac{\partial s_{22}}{\partial y}-P \frac{\partial \omega}{\partial x}=0
\end{aligned}
$$

These equations are solved as in the case of the initially stress-free medium. The displacement $u, v$ is expressed by the same relations (2.6) in terms of a function $\phi$. We may write a general solution in the form

$$
\begin{aligned}
& \phi=\frac{1}{l^{2}} f(l y) \sin l x \\
& s=L\left[\left(m+\frac{1}{2}-\frac{1}{2} k^{2}\right) f^{\prime}-f^{\prime \prime \prime}\right]
\end{aligned}
$$

with

$$
\begin{array}{cc}
m=\frac{2 M-L}{L} & k^{2}=\frac{L-P}{I} \\
M=N+\frac{1}{4} P & L=Q+\frac{1}{2} P
\end{array}
$$

The function $f$ satisfies the differential equation

$$
f^{\prime \prime \prime \prime}-2 m f^{\prime \prime}+k^{2} f=0
$$

where the prime denotes a differentiation with respect to $l y$. From the function $f$ it is possible to derive the displacements using the representation (2.12). The displacements are given as in equation (2.13) by

$$
\begin{aligned}
& l U(l y)=-f^{\prime} \\
& l V(l y)=f .
\end{aligned}
$$

Similarly the incremental stresses $s_{11} s_{22} s_{12}$ may be expressed in terms of $f$. Howcver for our purpose we shall evaluate the following expressions

$$
\begin{aligned}
& \iota_{11}=s_{11}-P e_{y y}=r(l y) \cos l x \\
& t_{22}=s_{22}=q(l y) \cos l x \\
& \Delta_{x y}=s_{12}+P e_{x y}=2 L e_{x y}=\tau(l y) \sin l x .
\end{aligned}
$$

The physical significance of these quantities is discussed in detail in the author's book [1] (pages $87,125,206$ ). The quantities $s_{22}$ and $\Delta_{x y}$ are respectively the normal and tangential stresses on a surface initially perpendicular to the $y$ axis. The quantity $t_{11}$ is the incremental normal stress per unit initial area initially perpendicular to the $x$ axis. In terms of 
$f$ we derive

$$
\begin{aligned}
& \frac{1}{L} r(l y)=-f^{\prime}-f^{\prime \prime \prime} \\
& \frac{1}{L} q(l y)=(2 m+1) f^{\prime}-f^{\prime \prime \prime} \\
& \frac{1}{L} \tau(l y)=-f-f^{\prime \prime} .
\end{aligned}
$$

An exponential solution $f=\exp (\beta l y)$ of the differential equation (4.6) is obtained when $\beta$ satisfies the characteristic equation

$$
\beta^{4}-2 m \beta^{2}+k^{2}=0
$$

Two of the roots may be written

$$
\begin{aligned}
& \beta_{1}=\left[m+\sqrt{ }\left(m^{2}-k^{2}\right)\right]^{\frac{1}{2}} \\
& \beta_{2}=\left[m-\sqrt{ }\left(m^{2}-k^{2}\right)\right]^{\frac{1}{2}} .
\end{aligned}
$$

The other two roots are then $-\beta_{1}$ and $-\beta_{2}$.

In the present analysis we shall again assume the inequality $(2.16)$, hence $m>1$. In this case

$$
m^{2}-k^{2}=m^{2}-1+\frac{P}{L}>0 .
$$

Therefore $\sqrt{ }\left(m^{2}-k^{2}\right)$ may be chosen as a positive real quantity. The value (4.11) of $\beta_{1}$ is real and may also be chosen as positive. The value of $\beta_{2}$ may be real or a pure imaginary. If

$$
P<L
$$

the value of $\beta_{2}$ is real and is then chosen positive. If

$$
P>L
$$

the value of $\beta_{2}$ is a pure imaginary and we may write it as

$$
\beta_{2}=i \xi
$$

where $\xi$ is a positive real quantity. The biquadratic (4.10) implies the following relations

$$
\beta_{1} \beta_{2}=k \quad \beta_{1}^{2}+\beta_{2}^{2}=2 m .
$$

When $k^{2}$ is negative $\beta_{2}$ is imaginary. In order to be consistent with equation (4.15) we choose for $k$ the value with a positive coefficient of $i$.

It is of interest to note the formal identity of equations (4.7) and (4.9) with the corresponding equations (2.13) for the medium initially free of stress. The general solution for $f$ in the case of initial stress is formally the same as expression (2.20) where the values of the roots $\beta_{1}$ and $\beta_{2}$ are now given by equations (4.11). 


\section{SKIN EFFECT OF SURFACE INSTABILITY}

An anisotropic incompressible half-space occupying the region $y<0$ is under the initial compressive stress $P$ parallel to the free surface $(y=0)$.

The author has shown $[1,3]$ that there is a critical value of the compression $P$ for which the surface is unstable. The critical value of $P$ is smaller than $L$. Hence, as pointed out in the previous section, the roots $\beta_{1}$ and $\beta_{2}$ are real and chosen positive.

A solution of the differential equation (4.6) is

$$
f=C_{1} f_{1}+C_{2} f_{2}
$$

where $f_{1}=\exp \left(\beta_{1} l y\right)$ and $f_{2}=\exp \left(\beta_{2} l y\right)$. This solution vanishes at $y=-\infty$. We choose the constants

$$
C_{1}=-\left(1+\beta_{2}^{2}\right) \quad C_{2}=1+\beta_{1}^{2} .
$$

Actually these constants may still contain a common arbitrary factor. This is a consequence of the fact that we are dealing with homogeneous boundary conditions and that solutions are valid with an arbitrary amplitude. For simplicity in the present analysis this factor is not included.

Substituting the values (5.1) (5.2) into the third of equations (4.9) we derive

$$
\frac{1}{L} \tau(l y)=\left(1+\beta_{1}^{2}\right)\left(1+\beta_{2}^{2}\right)\left(f_{1}-f_{2}\right) .
$$

Hence $\tau=0$ for $y=0$ and there is no tengential stress at the surface.

The same substitution of $f$ in the second of equations (4.9) after taking into account relations (4.16), yields

$$
\frac{1}{L} q(l y)=\left(1+\beta_{1}^{2}\right)^{2} \beta_{2} f_{2}-\beta_{1}\left(1+\beta_{2}^{2}\right)^{2} f_{1} .
$$

The normal stress $q$ vanishes at the surface $y=0$ if

$$
\left(1+\beta_{1}^{2}\right)^{2} \beta_{2}-\left(1+\beta_{2}^{2}\right)^{2} \beta_{1}=0 .
$$

This is the condition which determines the critical compression $P$ for instability of a free surface. Using relations (4.16) and eliminating the common factor $\beta_{1}-\beta_{2}$ we obtain condition (5.5) in the form

$$
2 k(m+1)+k^{2}-1=0 .
$$

This equation was obtained previously and discussed in detail by the author $[1,3]$. Finally we substitute the expression (5.1) for $f$ into the first of equations (4.9). We derive

$$
\frac{1}{L} r(l y)=\left(1+\beta_{1}^{2}\right)\left(1+\beta_{2}^{2}\right)\left(\beta_{1} f_{1}-\beta_{2} f_{2}\right) \text {. }
$$

As before consider the case of strong anisotropy, hence $N \gg Q, m \gg 1$. The instability condition (5.6) may be solved approximately for $k$. Hence

$$
k=\frac{1}{2(m+1)} \quad k \ll 1
$$


From the definition (4.5) of $k^{2}$ we derive the critical compressive stress

$$
P=L\left[1-\frac{1}{4(m+1)^{2}}\right]
$$

This value is only slightly smaller than $L$. Approximate values of the characteristic roots are obtained from equations (4.11) and (4.16). They are written

$$
\begin{aligned}
& \beta_{1}=\sqrt{ }(2 m) \\
& \beta_{2}=\frac{k}{\beta_{1}}=\frac{1}{2 \sqrt{ }(2 m)(m+1)} .
\end{aligned}
$$

Expressions (5.3) and (5.7) may be written approximately

$$
\begin{aligned}
& \frac{1}{L} \tau(l y)=\beta_{1}^{2}\left(f_{1}-f_{2}\right) \\
& \frac{1}{L} r(l y)=\beta_{1}^{3} f_{1} .
\end{aligned}
$$

The stress distribution as a function of depth is represented schematically in Fig. 2.

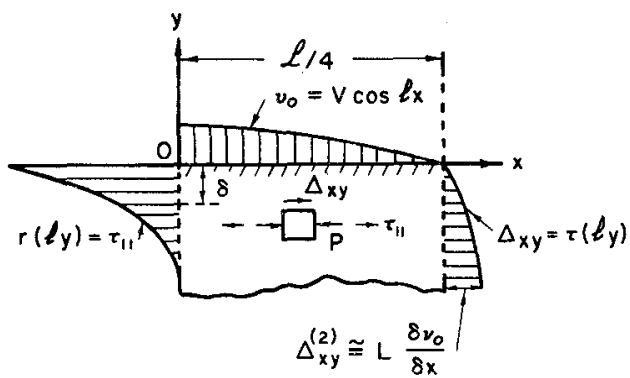

FIG. 2. Skin effect of surface instability under initial compressive stress $P$ with surface deflection $v_{0}=V \cos l x$. Plot shows distribution of incremental stresses $t_{11}=r(l y)$ at $x=0$ and $\Delta_{x y}=\tau(l y)$ at $x=\mathscr{L} / 4$. Values of $r(l y)$ and $\tau(l y)$ are respectively positive and negative. The sign is not indicated in the plot.

Since $\beta_{2}$ is quite small the function $f_{2}$ decays very slowly and may be replaced by unity near the surface. The term $f_{1}$ varies rapidly by a factor $1 / \mathrm{e}$ through a distance $\delta$ which represents a skin effect and is the same as in the previous equations (3.9) and (3.10). Hence we write approximately

$$
\delta \cong \frac{\mathscr{L}}{2 \pi \sqrt{(2 m)}}
$$

where $\mathscr{L}$ is the buckling wavelength. Putting

$$
\begin{aligned}
& \tau_{1}(l y)=L \beta_{1}^{2} f_{1} \\
& \tau_{2}(l y)=-L \beta_{1}^{2} f_{2}
\end{aligned}
$$


equations (5.11) become

$$
\begin{aligned}
& \tau(l y)=\tau_{1}(l y)+\tau_{2}(l y) \\
& r(l y)=\beta_{1} \tau_{1}(l y) .
\end{aligned}
$$

The second equation shows the same stress concentration factor $\beta_{1} \doteq \sqrt{ }(2 m)$ as in equation (3.15).

The instability condition (5.5) is independent of the wavelength. Assuming a vertical deflection $v=V \cos l x$ and applying the second of equations (4.7) we derive approximately

$$
l V(l y)=\beta_{1}^{2} f_{2}=-\frac{1}{L} \tau_{2}(l y) .
$$

Hence the tangential stress $\Delta_{x y}^{(2)}$ corresponding to the term $\tau_{2}(l y)$ may be written

$$
\Lambda_{x y}^{(2)}=\tau_{2}(l y) \sin l x=I \frac{\partial v}{\partial x} .
$$

This expression varies very slowly with depth and may be assumed independent of $y$ within the skin thickness. As a consequence we derive the approximate valuc

$$
\Delta_{x y}^{(2)}=\tau_{2}(0) \sin l x=L \frac{\mathrm{d} v_{0}}{\mathrm{~d} x}
$$

where $v_{0}$ is the surface deflection. Since $\tau_{1}+\tau_{2}=0$ at the surface we also derive

$$
\tau_{1}(0) \sin l x=-L \frac{\mathrm{d} v_{0}}{\mathrm{~d} x}
$$

Numerically the assumption of large anisotropy turns out to be already appreciable for values of $M / L$ which are not large. For example if $M / L=4.5$ we derive the same approximate values $\beta_{1} \cong \sqrt{ }(2 m)=4$ and $\beta_{2} \cong 1 / \sqrt{ }(2 m)=1 / 4$ as in equation (3.4).

\section{INTERNAL BUCKLING WITH SKIN EFFECT AT A FREE BOUNDARY}

Internal buckling occurs for $P>L$. This phenomenon was analyzed in detail by the author for an indefinite or confined medium $[1,2]$. It was shown that there are two types of internal buckling in anisotropic media which we have referred to as internal buckling of the first and second kind.

We shall assume as before that $m>1$. From previous results it follows that only buckling of the first kind is possible in this case. The root $\beta_{1}$ is real while $\beta_{2}=i \xi$ is a pure imaginary. Relations (4.16) for the roots may be written

$$
\begin{gathered}
\beta_{1} \xi=k^{\prime} \quad \beta_{1}^{2}-\xi^{2}=2 m \\
k^{\prime}=\frac{k}{i}=\sqrt{ }\left(\frac{P}{L}-1\right) .
\end{gathered}
$$

A solution of the differential equation (4.6) corresponding to the characteristic roots 
$\pm \beta_{2}= \pm i \xi$ is

$$
f_{3}=\sin \xi l y \text {. }
$$

We substitute this value for $f$ in the second and third of equations (4.9) after taking into account the second of relations (6.1), we derive

$$
\begin{aligned}
& \frac{1}{L} q_{3}(l y)=\left(1+\beta_{1}^{2}\right) \xi \cos \xi l y \\
& \frac{1}{L} \tau_{3}(l y)=-\left(1-\xi^{2}\right) \sin \xi l y .
\end{aligned}
$$

The subscript 3 is used to indicate that these values are those associated with the solution $f_{3}$. The displacements are obtained from equations (4.7)

$$
\begin{gathered}
l U_{3}(l y)=-\xi \cos \xi l y \\
l V_{3}(l y)=\sin \xi l y .
\end{gathered}
$$

The tangential stress $\tau$ and the displacement $V$ vanish for $y=0$. They also vanish for $y=h$ provided

$$
\xi=\frac{\pi}{l h}=\frac{\mathscr{L}}{2 h}
$$

where $\mathscr{L}$ is the wavelength along the $x$ direction.

Hence the solution (6.2) represents the internal buckling of a medium subject to a compression $P$ and confined between two rigid frictionless parallel walls separated by a distance $h$ as illustrated in Fig. 3(a). Since $i \xi$ is a characteristic root, equation (4.10) is verified by substituting $\beta=i \xi$. If we solve the resulting equation for $P$ we obtain

$$
P=L+2(2 M-L) \xi^{2}+L \xi^{4} .
$$

This is the value of the compression $P$ required to maintain an internal buckling mode of wavelength $\mathscr{L}=2 h \xi$ as shown by relation (6.5).

Equation (6.6) coincides with the result obtained previously in a detailed analysis of internal buckling of a confined medium $[1,2]$. As pointed out these buckling modes are metastable. For a given value of $P>L$ there is a continuous range of unstable modes with wavelengths between zero and the value corresponding to equation (6.6). As before we assume the anisotropy to be large. Hence

$$
m=\frac{2 M-L}{L} \gg 1 \text {. }
$$

(a)

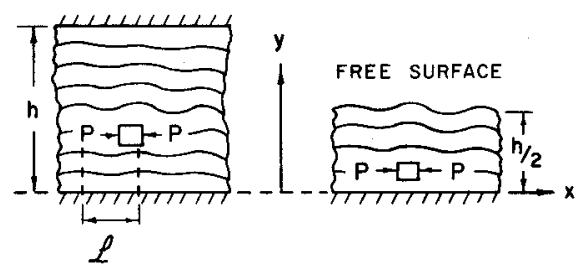

FIG 3. (a) Internal buckling of a confined medium under initial compressive stress $P$. (b) Internal buckling of the same medium with a free surface at $y=h / 2$. 
Approximate values of $\beta_{1}$ and $\xi$ in this case are

$$
\begin{aligned}
\beta_{1} & =\sqrt{ }(2 m) \\
\xi & =\frac{k^{\prime}}{\beta_{1}}=\sqrt{ }\left[\frac{1}{2 m}\left(\frac{P}{L}-1\right)\right] .
\end{aligned}
$$

Furthermore it is assumed that $P$ is only moderately larger than the lower critical value L. Hence

$$
\xi \ll 1
$$

Under these conditions we derive from (6.3) the approximate values

$$
\begin{aligned}
& \frac{1}{L} q_{3}(l y)=\beta_{1}^{2} \xi \cos \xi l y \\
& \frac{1}{L} \tau_{3}(l y)=-\sin \xi l y .
\end{aligned}
$$

Using equations (6.4) and (6.10) we obtain

$$
\begin{aligned}
& q_{3}=-\tau_{3} \beta_{1}^{2} \xi \cot \xi l y \\
& \tau_{3}=-L l V_{3} .
\end{aligned}
$$

Introducing the definitions (2.12) and (4.8) the last equation may be written

$$
\Delta_{x y}^{(3)}=L \frac{\partial v}{\partial x}
$$

Hence the tangential stress $\Delta_{x y}^{(3)}$ associated with the solution $f_{3}$ is determined approximately by the horizontal derivative of the vertical displacement $v$.

We now assume the presence of a free surface at $y=h / 2$ as illustrated in Fig. 3(b). In order to cancel the stresses $q_{3}, \tau_{3}$ at the free surface we must add a solution corresponding to the characteristic root $\beta_{1}$. Such a solution is

$$
C_{1} f_{1}=C_{1} \mathrm{e}^{\beta_{1} l y}
$$

with an undetermined constant $C_{1}$. The stresses $r_{1}, q_{1}, \tau_{1}$ generated by this solution are obtained by applying equations (4.9). When $\beta_{1}$ is large their approximate values are given by

$$
\begin{aligned}
& \frac{1}{L} r_{1}(l y)=-C_{1} \beta_{1}^{3} f_{1} \\
& \frac{1}{L} q_{1}(l y)=C_{1} \beta_{1} f_{1} \\
& \frac{1}{L} \tau_{1}(l y)=-C_{1} \beta_{1}^{2} f_{1} .
\end{aligned}
$$

By superposition of the solutions $f_{3}$ and $f_{1}$ the total normal and tangential stresses at the free surface $y=h / 2$ are made to vanish by choosing the constant $C_{1}$ such that

$$
\begin{aligned}
\tau_{3}\left(\frac{l h}{2}\right)+\tau_{1}\left(\frac{l h}{2}\right) & =0 \\
q_{3}\left(\frac{l h}{2}\right)+q_{1}\left(\frac{l h}{2}\right) & =0 .
\end{aligned}
$$


Elimination of $C_{1} \exp \left(\beta_{1} l h / 2\right)$ between these two equations yields

$$
\tan \xi \frac{l h}{2}=\beta_{1}^{3} \xi
$$

Referring to the values. (6.8) of $\xi$ and $\beta_{1}$ we see that equation (6.16) determines the compression $P$ required to maintain a buckling mode of given wavelength $\mathscr{L}=2 \pi / l$ in the presence of a free surface.

The foregoing analysis shows that the influence of the free surface is embodied essentially in the term $C_{1} f_{1}$ given by expression (6.13). It decays very fast with the distance from the free surface and gives rise to a skin effect with the same skin thickness $\delta$ as in equation (3.10)

$$
\delta=\frac{\mathscr{L}}{2 \pi \sqrt{ }(2 m)} .
$$

The tangential stress is the sum of two terms. One of these terms is $\tau_{3}(l y)$ as given by equation (6.10). It varies slowly with $y$ and is almost constant within the skin thickness. The other term according to equations (6.14) may be written

$$
\tau_{1}(l y)=\tau_{1}\left(\frac{l h}{2}\right) \mathrm{e}^{\beta_{1} l[y-(h / 2)]}
$$

and decays very fast within the skin thickness.

Equations (6.15) also show that the tangential stress $\tau_{1}$ is associated with a normal stress $r_{1}$ parallel to the surface and equal to

$$
r_{1}(l y)=\beta_{1} \tau_{1}(l y) \text {. }
$$

This relation exhibits the same stress concentration factor $\beta_{1}$ as in the foregoing equations (3.15) and (5.14).

The solution $f_{3}$ contributes only a small value $r_{3}(l y)$ to the total component $r(l y)$ and may be neglected. Hence the total stresses $\tau$ and $q$ within the skin thickness are distributed as in Fig. 2 for the case of surface instability.

Note that according to equation (6.12) the approximate value of the stress component $\tau_{1}(l h / 2)=-\tau_{3}(l h / 2)$ at the surface is given by .

$$
\tau_{1}\left(\frac{l h}{2}\right) \sin l x=-L \frac{\mathrm{d} v_{0}}{\mathrm{~d} x}
$$

where $\mathrm{d} v_{0} / \mathrm{d} x$ is the surface slope. A similar result was derived for surface instability as shown by equation (5.18). Let us also examine the significance of the buckling condition (6.16) and consider the case where $\beta_{1}^{3} \xi$ is large. This implies a large value of $\tan (\xi l h / 2)$ and we may write approximately

$$
\xi l h=\pi .
$$

The value of $\xi$ thus obtained coincides with the value (6.5). Hence in this case the buckling mode and the compression $P$ required for buckling with a given wavelength $\mathscr{L}$ is approximately the same as for a confined medium of twice the total thickness. The only specific feature due to the free surface occurs in a thin layer of thickness $\delta$ near the surface.

The range of validity of the assumptions may be illustrated by considering the 
numerical values

$$
\xi=\frac{\mathscr{L}}{2 h}=\frac{1}{4} \quad \frac{M}{L}=4 \cdot 5
$$

hence

$$
\beta_{1}=\sqrt{ }(2 m)=4 .
$$

In this case $\beta_{1}^{3} \xi=16$ and equation (6.16) is approximately verified by putting $\xi l h=\pi$ in accordance with the value (6.21). Hence the critical compressive stress is not affected significantly by the presence of a free surface and is very close to the value (6.6) derived for a confined medium. The foregoing numerical values also imply $P=2 L$. We note that the assumptions we have introduced are already verified if $m$ is not actually very large.

Relation to the theory of plate stability

Examination of Fig. 3(b) shows that the problem of internal buckling in the presence of a free surface and a frictionless rigid boundary is identical to the case of instability of a thick plate for deformations symmetric with respect to the plane of symmetry. The plane of symmetry corresponds to the rigid frictionless boundary in Fig. 3(b).

The theory of plate stability under a compression $P$ parallel to the faces has been developed in detail by the author $[1,4,5]$. The condition for occurrence of a symmetric buckling mode in a plate of thickness $h$ was found to be

$$
R_{1} z_{1}^{\prime}-R_{2} z_{2}^{\prime}=0 \text {. }
$$

This equation is given on page 328 in the author's book [1]. For static buckling and an incompressible material we may write

$$
\begin{array}{ll}
R_{1}=\left(1+\beta_{1}^{2}\right)^{2} & R_{2}=\left(1+\beta_{2}^{2}\right)^{2} \\
z_{1}^{\prime}=\frac{1}{\beta_{1}} \tanh \left(\beta_{1} \frac{l h}{2}\right) & z_{2}^{\prime}=\frac{1}{\beta_{2}} \tanh \left(\beta_{2} \frac{l h}{2}\right) .
\end{array}
$$

When $\beta_{2}=i \xi$ and for large real values of $\beta_{1}$ equation (6.24) becomes *

$$
\beta_{1}^{3}-\frac{1}{\xi} \tan \xi \frac{l h}{2}=0
$$

which is the same as the approximate buckling condition (6.16).

\section{INTERNAL BUCKLING WITH SKIN EHFECT AT A RIGID ADHERING BOUNDARY}

Consider the internal buckling illustrated in Fig. 3(a) and assume that perfect adherence occurs at the upper rigid boundary $y=h$. As we shall see the effect of the adherence is to produce a disturbance confined near the boundary.

The initial stress is a uniform compression $P$ parallel to the boundary. In the present case such a state of stress could be produced by deforming the medium homogeneously with a variable gap between frictionless rigid boundaries after which the upper boundary is made to adhere to the deformed medium. Another procedure would be to use as upper 
boundary a very strong medium which is deformed homogeneously under very high stress by the same amount as the confined medium.

The internal buckling solution $f_{3}$ generates at the upper boundary displacements $U_{3}(l h)$ and $V_{3}(l h)$ obtained by substituting $y=h$ into equations (6.4),

$$
\begin{gathered}
l U_{3}(l h)=-\xi \cos \xi h \\
l V_{3}(l h)=\sin \xi l h .
\end{gathered}
$$

Perfect adherence requires cancellation of these displacements by adding the solution $C_{1} f_{1}=C_{1} \exp \beta_{1} l y$ of equation (6.13). By applying equations (4.7) the displacements $U_{1}, V_{1}$ associated with the solution $C_{1} f_{1}$ are found to be given by

$$
\begin{aligned}
l U_{1}(l h) & =-C_{1} \beta_{1} \exp \beta_{1} l h \\
l V_{1}(l h) & =C_{1} \exp \beta_{1} l h .
\end{aligned}
$$

Perfect adherence requires

$$
\begin{array}{r}
U_{3}(l h)+U_{1}(l h)=0 \\
V_{3}(l h)+V_{1}(l h)=0 .
\end{array}
$$

Elimination of $C_{1} \exp \left(\beta_{1} l h\right)$ between these two equations yields the approximate buckling condition

$$
\tan \xi l h=\xi / \beta_{1}
$$

Assuming the same numerical values (6.22) and (6.23) as in the previous case, equation (7.4) is approximately verified by $\xi l h=\pi$ which coincides with the values (6.5) and (6.21). Hence the buckling condition is not significantly affected by the adherence.

The term $C_{1} f_{1}$ in the total solution represents the disturbance introduced by the adherence. Its effect is confined near the boundary with a skin effect of thickness $\delta$ given as before by equation (6.17).

The stress disturbance within the skin thickness is given by equations (6.14). Relation (6.19) with the stress concentration factor $\beta_{1}$ is also applicable.

\section{SKIN EFFECT OF SURFACE WAVES INCLUDING THE INFLUENCE OF INITIAL STRESS}

A skin effect of a type entirely similar to that analyzed above occurs for surface waves in anisotropic solids. This can be seen immediately by applying the equations derived by the author for the dynamics of anisotropic media under initial stress $[1,4]$. The results are of course valid for the particular case of the initially stress-free medium.

The same solutions as used in Section 5 for surface instability are applicable to dynamics with two characteristic roots $\beta_{1}$ and $\beta_{2}$. The roots in this case depend on the circular frequency $\alpha$ and the density $\rho$. For example for an incompressible solid they are obtained from the same equation (4.10) where the coefficients as derived by the author 
$[1,4]$ are

$$
\begin{aligned}
2 m & =\frac{1}{L}\left(4 M-2 L-\frac{\alpha^{2} \rho}{l^{2}}\right) \\
k^{2} & =\frac{1}{L}\left(L-P-\frac{\alpha^{2} \rho}{l^{2}}\right) .
\end{aligned}
$$

Under the assumptions

$$
m \gg 1 \quad 0<k^{2}<1
$$

the roots $\beta_{1}$ and $\beta_{2}$ are real, with a large value for $\beta_{1}$ and a small value for $\beta_{2}$. Proceeding as in Section 5 it can be seen that exactly the same skin effect is obtained as for surface instability.

\section{Skin effect for compressible solids}

In the preceding analysis it was assumed for analytical simplicity that the solid is incompressible. The skin effect also occurs for strongly anisotropic compressible solids and may easily be evaluated numerically by applying the general equations derived by the author for the dynamics of anisotropic compressible media under initial stress $[1,4]$.

The skin effect features are obtained in terms of characteristic roots $\beta_{1}$ and $\beta_{2}$ respectively large and small and are essentially the same as in the various cases considered in the foregoing analysis for an incompressible solid. Values of the roots $\beta_{1}$ and $\beta_{2}$ are expressed by the same formulas (4.11) in terms of the two basic parameters $m$ and $k^{2}$. However in this case these parameters depends on the various elastic moduli describing the elastic properties of the compressible anisotropic medium. The complete expressions for $m$ and $k^{2}$ in this case will be found in the author's previous book and paper $[1,4]$.

\section{SKIN EFFECT IN ANISOTROPIC VISCOELASTICITY}

From the principle of viscoelastic correspondence $[6,7]$ we may conclude that a skin effect will also occur in a strongly anisotropic viscoelastic material. Of particular interest is the case of a purely viscous anisotropic medium. For example the analysis of Section 3 is immediately applicable to this case since $N / Q$ is replaced by the ratio of two viscosity coefficients and the characteristic roots remain algebraic quantities independent of any operator. The skin thickness is given by the same expression (3.10). Applicability of viscoelastic correspondence to such materials including the effect of initial stress was already indicated in earlier work [7] and has been discussed in detail more recently [8].

\section{REFERENCES}

[1] M. A. Biot, Mechanics of Incremental Deformations. Wiley (1965).

[2] M. A. BIot, Internal buckling under initial stress in finite elasticity. Proc. R. Soc. A273, 306 (1963)

[3] M. A. B1oт, Surface instability in finite anisotropic elasticity under initial stress. Proc. R. Soc. A273, 329 (1963).

[4] M. A. Bıot, Continuum dynamics of elastic plates and multilayered solids under initial stress. J. Math. Mech. 12, 793 (1963).

[5] M. A. BIOT, Theory of stability of multilayered continua in finite anisotropic elasticity. J. Franklin Inst. 276, 128 (1963). 
[6] M. A. Biot, Dynamics of viscoelastic anisotropic media. Proc. 2nd Midwestern Conference on Solid Mechanics Research Series No. 129, Engineering Experiment Station, Purdue University, Lafayette, Ind., pp. 94-108, 1955.

[7] M. A. Biot, Variational and Lagrangian methods in viscoelasticity, deformation and flow of solids. (IUTAM Colloquiem, Madrid 1955), pp. 251-263. Springer (1956).

[8] M. A. Bior, Internal instability of anisotropic viscous and viscoelastic media under initial stress. J. Franklin Inst. 279, 65 (1965).

(Received 21 February 1966)

Résumé-Il est démontré que dans la mécanique solide un effet pelliculaire est relié à l'anisotropie. Près d'une surface libre ou surface de discontinuité, certaines composantes du champ des tensions varient rapidement de zéro à un maximum à l'intérieur d'une pellicule mince. L'épaisseur de cette pellicule tend à fondre pour une anisotropie allant en augmentant. Une concentration de tension a également lieu, par laquelle certaines composantes dés tensions sont amplifiées á l'intérieur de l'épaisseur de la pellicule. L'analyse est entreprise pour un milieu avec ou sans contrainte initiale et comprend les cas d'instabilité de surface, de flambage interne et de propagation d'ondes de surface. Les résultats présentés dans le contexte de la théorie de l'élasticité sont en général valables pour les milieux viscoélastiques selon et principe de la correspondance. Les résultats sont également applicables à des materiaux composés à couches multiples ou fibreux qui, en moyenne, se comportent approximativement comme des milieux continus anisotropes.

Zusammenfassung - Es wird gezeigt, dass in der Festkörpermechanik Hauteffekt und Anisotropie miteinander vebuden sind. In der Nähe einer freien Oberfläche oder einer Diskontinuitäts-Oberfläche ändern sich gewisse Komponenten des Spannungsfeldes in der dünnen Haut schnell zwischen Null und dem Maximalwert. Die Dicke dieser Haut verschwindet ganz wenn die Anisotropie zunimmt. Ferner wird die Spannung auch konzentriert indem gewisse Spannungs-Komponenten innerhalb der Hautdicke verstärkt werden. Die Analyse wird für Material mit oder ohne Anfangsspannung durchgeführt einschliesslich der Fälle mit Instabilität der Oberfläche, mit innerem Knicken und Oberflächen-Wellenausbreitung. Die Resultate werden in Rahmen der Elastizitatstheorie gegeben, dem Korrespondenzprinzip entsprechend gelten sie auch für viskoelastische Materialen; sowie auch für mehrschichtige und faserige Materialen, die sich allgemein und ungefähr wie kontinuierliche anisotropische Materialen benehmen.

Абстракт-Показывается, что в механике твёрдых тел скин-эффект связан с анизотропией. Вблизи свободной поверхности или поверхности разрыва некоторые составные части поля напряжения быстро изменяются с нуля до максимума в пределах тонкого наружного слоя. Толщина этого наружного слоя стремится к нулю с увеличивающейся анизотропией. Концентрация напряжения получается также поскольку некоторые компоненты напряжения усиливаются в пределах толщины наружного слоя. Анализ проводится для среды (материала) с начальным или без начального напряжения и включает случай неустойчивости поверхности, внутреннего выпучивания и распространения поверхностной волны. Результаты представлены в контексте теории упругости и в общем и целом ценны для вяэко-зластичной среды, согласно принципу соответствия. Результаты также применяются в случае многослойных или волокнистых сложных материалов, которые, в среднем, ведут себя, приблизительно, как анизотропная непрерывная среда. 